\title{
VRH investigation of polyaniline-multiwalled carbon nanotube nanocomposite network
}

\author{
AMIN IMANI and GHOLAMALI FARZI* \\ Department of Materials and Polymer Engineering, Faculty of Engineering, Hakim Sabzevari University, \\ P.O. Box 397, Sabzevar 009851, Iran
}

MS received 19 September 2014; accepted 27 March 2015

\begin{abstract}
Polyaniline and polyaniline/multi-walled carbon nanotube (PAni/MWCNT) nanocomposites were synthesized by in-situ chemical oxidative polymerization of aniline. Ammonium peroxydisulphate and p-toluenesulphonic acid were used as an initiator and surfactant dopant, respectively. The molar ratio of monomer unit to initiator and dopant was 1:1:1 and the percentage of MWCNT in PAni varied from 1 to 10 wt \%. The structure of the resulting nanocomposite was characterized by scanning electron microscopy, X-ray diffraction and Fourier transform infrared spectroscopy. The effects of MWCNT concentration on the electrical properties of the resulting nanocomposites were studied at temperatures between 90 and $300 \mathrm{~K}$. Conductivity increases with the combination of MWCNT in the PAni environment. The strong coupling between the MWCNT and the PAni chains enhances the average localization length and hence conductivity increases for the nanocomposites.
\end{abstract}

Keywords. Polyaniline; MWCNT; nanocomposites; VRH model; oxidative polymerization.

\section{Introduction}

Conducting polymers appear to be one of the most significant group of materials with an enormous field of applications from primarily passive materials such as coatings and containers to active materials with useful optical, electronic and energy storage properties. They can be synthesized either by reduction or oxidation reaction, the so-called doping process. Also, existence of localized electronic states of energies less than the band gap arising from changes in local bond order, containing the formation of solitons, polarons and bipolarons, has led to the chance for new types of charge conduction in these polymers. ${ }^{1-5}$ In spite of strong electronphonon coupling in conducting polymers, an amazing large range of conductivities has been covered by doping. Their conductivity ranges from the highly insulating values $10^{-6} \mathrm{~S} \mathrm{~cm}^{-1}$ to highly metallic ones $10^{5} \mathrm{~S} \mathrm{~cm}^{-1}$ depending on the doping concentration. ${ }^{6}$ Conducting polymers are suitable for many potential applications in electronic and electrochromic devices, corrosion protection, rechargeable and lightweight batteries, drug delivery, membrane separation, supercapacitors and sensors owing to their wide ranges of conductivities. Among several conducting polymers, polyaniline (PAni) has been known for more than 150 years. ${ }^{7}$ It has drawn a lot of interest from investigators because of its exclusive properties such as high conductivity, simple methods of synthesis and easy formulation as a composite matrix and stability.

PAni provides useful properties, but to adjust the polymer aspects and attain excellent properties, it has been used in

\footnotetext{
*Author for correspondence (alifarzi@yahoo.com)
}

composites structure by merging with nanofillers such as carbon nanotubes (CNTs). The large specific area and aspect ratio of multiwalled carbon nanotubes (MWCNTs) have made them well-organized as conductive fillers in conducting polymers. $^{8}$

There has been much attention on the temperature and composition behaviour of the conductivity of PAni and in the mechanism of its conduction. ${ }^{3}$ PAni is recognized to experience a metal-insulator transition as a function of doping. Conduction data in PAni at low fields in this regime are usually discussed within the framework of the standard Mott variable-range hopping (VRH) model in the presence of Coulomb interactions at low temperatures. ${ }^{6}$

In this work, PAni and PAni/MWCNT nanocomposites have been synthesized using chemical in-situ oxidative polymerization. Based on Fourier transform infrared (FT-IR) spectroscopy, X-ray diffraction (XRD) and scanning electron microscopy (SEM) analysis, amorphous PAni completely coats MWCNT. The effect of MWCNT on the electrical properties and conductivity temperature dependence of nanocomposites were discussed. The conductivity carrier concentration and mobility of nanocomposites were discussed using the VRH model, from 90 to $300 \mathrm{~K}$.

\section{Experimental}

\subsection{Materials}

MW-CNTs (with 10-20 nm diameter, $30 \mu \mathrm{m}$ length and density $2.1 \mathrm{~g} \mathrm{~cm}^{-3}$ ) made by chemical vapour deposition were provided by the Petroleum Research Institute of 
Iran. The purity of the MWCNTs was reported to be $98 \%$. Aniline was purchased from Merck and purified under low pressure before use. Ammonium peroxydisulphate (APS) and p-toluenesulphonic acid (p-TSA) were obtained from Merck and used in our experiment as-received. All other chemicals and solvents were of analytical grade and were used without any further purification.

\subsection{Synthesis of PAni and PAni/MWCNT nanocomposites}

The nanocomposites of PAni/MWCNT were synthesized by chemical in-situ oxidation polymerization. The molar ratio of monomer/dopant/initiator was kept constant by $1: 1: 1$. In a typical manner, $0.014 \mathrm{~mol}$ of APS was dissolved in $75 \mathrm{ml}$ distilled water and stirred for $10 \mathrm{~min}$. A solution of p-TSA in water $(0.014 \mathrm{~mol}$ in $25 \mathrm{ml}$ water) was added to the aqueous solution of APS and stirred for $5 \mathrm{~min}$. Aniline $(0.014 \mathrm{~mol})$ was mixed with $50 \mathrm{ml}$ water and then proportional amount of MWCNT was added to the aniline solution and ultrasonicated for $15 \mathrm{~min}$. Later, mixture was added a bit by bit to the vessel containing APS/p-TSA solution and stirred for about $5 \mathrm{~h}$ at room temperature. The black sediment was washed with ethanol and distilled water consequently until the filtrate was colourless. Then, the specimens were dried under vacuum at $70^{\circ} \mathrm{C}$ for $48 \mathrm{~h}$. Several PAni/MWCNT nanocomposites were synthesized with different weight percentages of MWCNT. For assessment, PAni was also synthesized in a similar procedure and used as reference.

\section{Results and discussion}

To characterize the chemical structure of synthesized PAni/MWCNT nanocomposites, their FT-IR spectra were obtained. Figure 1 indicates the FT-IR spectra of these

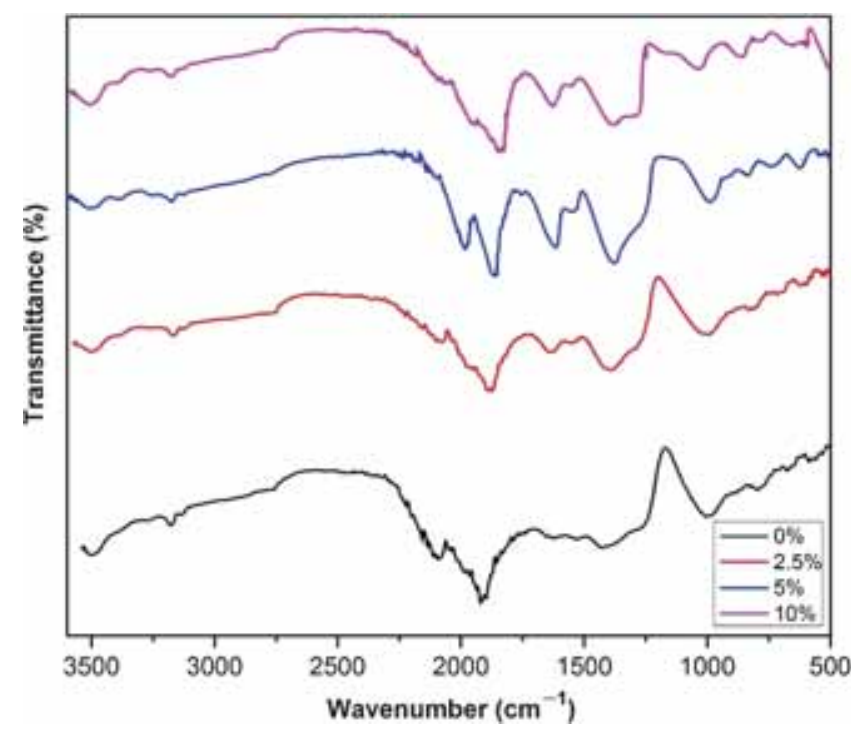

Figure 1. FT-IR spectra of pure PAni and PAni/MWCNT nanocomposites. nanocomposites. These spectra were attained with $\mathrm{KBr}$ in the pellet form. As can be seen, the spectra of nanocomposites are similar to that of the pure PAni. This figure shows peaks located at around $\sim 3225 \mathrm{~cm}^{-1}$ that are attributable to the $\mathrm{N}-\mathrm{H}$ stretching vibration. The bands located at $\sim 1580$ and $\sim 1499 \mathrm{~cm}^{-1}$ are related to the stretching vibration of quinoid and benzenoid rings, respectively. The peak at $\sim 1317 \mathrm{~cm}^{-1}$ can be corresponded to the $\mathrm{C}-\mathrm{N}$ type, whereas the peak at $\sim 1161 \mathrm{~cm}^{-1}$ is the characteristic bands of stretching vibration of quinoid; the bands appearing at $\sim 812 \mathrm{~cm}^{-1}$ are assigned to an aromatic $\mathrm{C}-\mathrm{H}$ bending vibration. ${ }^{9}$ When the bands were associated to the pure PAni, it can be seen that the peak's position of the PAni/MWCNT nanocomposites has a tiny shift to high wavenumber. It was due to the interaction between PAni and MWCNT. ${ }^{4,8}$ Also, it could be recognized by the enlarged influence of the charge-transfer consequence between the polymer chains and MWCNT. These statistics showing the presence of MWCNT in oxidative polymerization procedure indicates PAni in nanocomposites exhibiting a higher oxidation degree in comparison with pure PAni. ${ }^{10}$

The crystallographic arrangements of PAni and PAni/MWCNT nanocomposites are characterized using XRD measurements. As can be seen in figure 2, the nanocomposites were found to be amorphous that is in accordance with the findings of other researchers. ${ }^{11-13} \mathrm{X}$-ray patterns of MWCNT can be found elsewhere and there is sharp and high intensity diffraction peak existing at $2 \theta=26^{\circ}$ and two lower intensity peaks at $2 \theta=45^{\circ}$ and $62^{\circ},{ }^{14,15}$ which are pointed to the diffraction signature of the distance between the walls and the interwall spacing. ${ }^{8}$ In the case of PAni, peaks seem near $2 \theta=20^{\circ}$ and $26^{\circ}$, which are attributed to the periodicity parallel and perpendicular to the polymer chains. ${ }^{9}, 14$ For PAni/MWCNT nanocomposites, the XRD spectra show mutually both of the characteristic peaks of PAni and that of

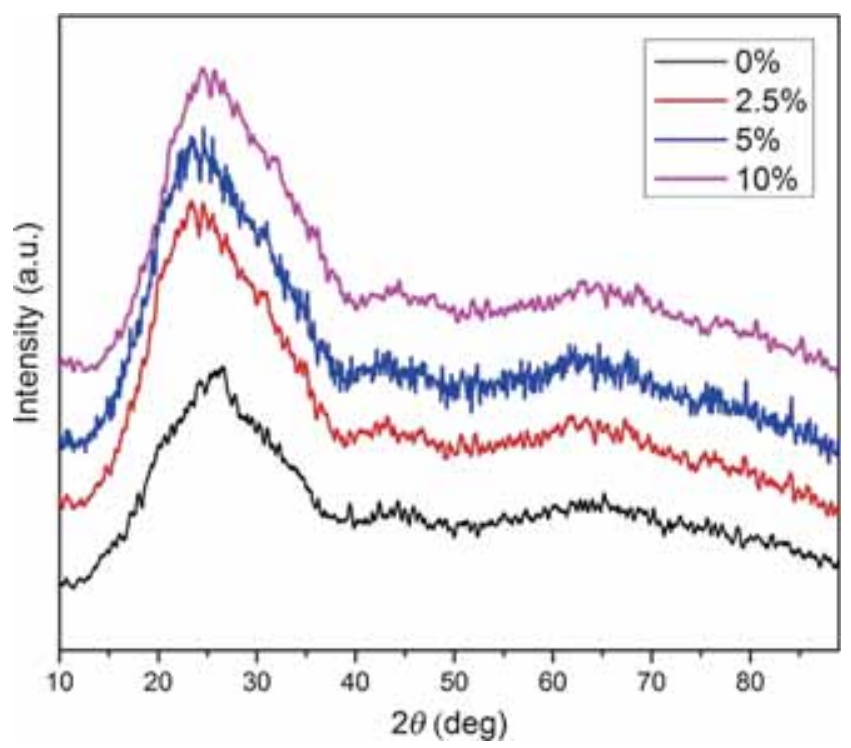

Figure 2. XRD patterns of PAni and PAni/MWCNT nanocomposites. 
MWCNT, displaying the attendance of MWCNT in PAni. In the nanocomposites spectra, the peak at $2 \theta=20^{\circ}$ has no obvious change, nevertheless the peak at $2 \theta=26^{\circ}$ is steadily raised with the increase of MWCNT weight percent.

Figure 3 exhibits the SEM micrograph of (a) PAni and (b) PAni/(5\%) MWCNT nanocomposites. The particle size of PAni found to be around 150-300 nm with orbicular morphology. As indicated by the SEM images, PAni presents a classically cauliflower-like morphology while the nanocomposite with 5\% MWCNT indicates nearly spherical aggregate and collective particles with some unpackaged morphology. The particle size of nanocomposite is smaller than that of pure PAni, it seems that MWCNT improve stability of PAni, and this may affect the morphology of PAni particles. Although XRD alignments specify the presence of MWCNT in the nanocomposite, the MWCNTs are obscured by means of SEM image.

For electrical property studies and measuring the conductivity, PAni and PAni/MWCNT nanocomposites were pressed into cylinder tablet formed under $10 \mathrm{MPa}$ pressure. The conductivity was measured at room temperature by a DC voltage/current gauge with the four-probe standard method. The conductivity values vs. MWCNT weight fraction are shown in figure 4 . As can be observed from the graph the conductivity of the PAni/MWCNT nanocomposites was raised by increasing the content of MWCNT related to that of pure PAni. The structure of nanocomposite (interaction between PAni and MWCNT) could be responsible for the improving conductivity. Also, large surface area of MWCNT may act as conducting link and connecting polymer spheres and increases effective penetration. ${ }^{16,17}$

In mathematics, percolation theory explains the performance of connected clusters in a random graph. ${ }^{18}$ In order to obtain a realizing to the conductivity percolation threshold, a mathematical function was fitted and then plotted on the experimental values. The percolation threshold role demonstrates as ${ }^{8,19,20}$

$$
\sigma=\sigma_{0}\left(P-P_{\mathrm{c}}\right)^{t},
$$

where $P, P_{\mathrm{c}}^{\prime}$ and $t$ are the weight fractions of MWCNT in the nanocomposite, the percolation threshold and the percolation exponent (it has a theoretical value of 2 for percolation), respectively. $\sigma_{0}$ is the scaling factor comparable

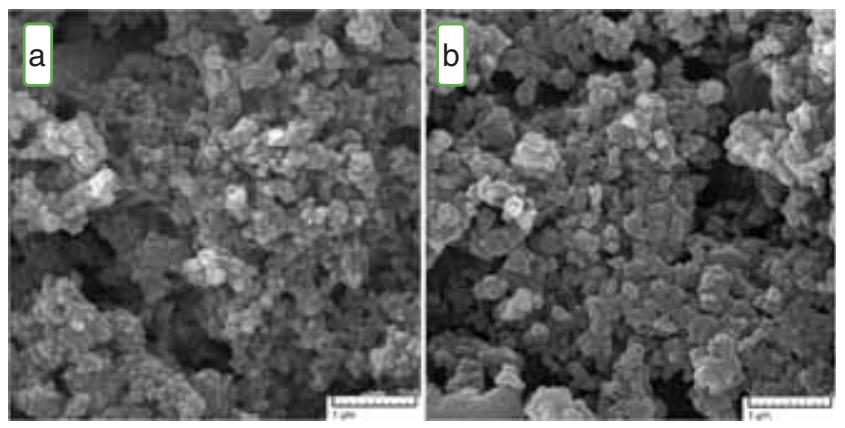

Figure 3. SEM images of (a) PAni and (b) PAni/MWCNT (5\%). to the effective bulk conductivity of MWCNT (i.e., at $\left.P=1, \sigma=\sigma_{\mathrm{MWCNT}}\right)$. Table 1 indicates the extracted values from conductivity curve. As can be deduced obviously, the value of $t=2.21$ confirmed that there is a good agreement between experimental and theoretical values (about $10 \%$ error). The threshold value for PAni/MWCNT was calculated to be around $4.2 \%$. It is clear that below the percolation threshold, there is less improvement in the conductivity value than above it. For values much greater than the percolation threshold, the conductivity was affected by the infinite value.

Conductivity variation follows Arrhenius law and obeys the following equation ${ }^{21}$

$$
\sigma(T)-\sigma_{\mathrm{c}} \exp \left[-\frac{E_{\mathrm{F}}-E_{\mathrm{C}}}{k_{\mathrm{B}}} T\right],
$$

where $E_{\mathrm{F}}, E_{\mathrm{C}}$ and $\sigma_{\mathrm{c}}$ are the Fermi energy, mobility edge and conductivity at the mobility edge, respectively. Nevertheless, researchers show that the Arrhenius model is not fully applicable to explaining the conductivity apparatus as in the case of normal semiconductors. There are other models which well known to explain the conductivity variations of conducting polymers, but it has been observed that for the lowtemperature range, the conductivity investigation are best demonstrated by VRH model. ${ }^{22,23}$ This model is appropriate for clarifying the temperature dependence of dc conductivity. The mechanism is founded on the impression that carriers tend to hop greater distances to sites which lie energetically closer rather than to their closest neighbours. ${ }^{6}$ In addition, an electron near the Fermi level skips among localized states

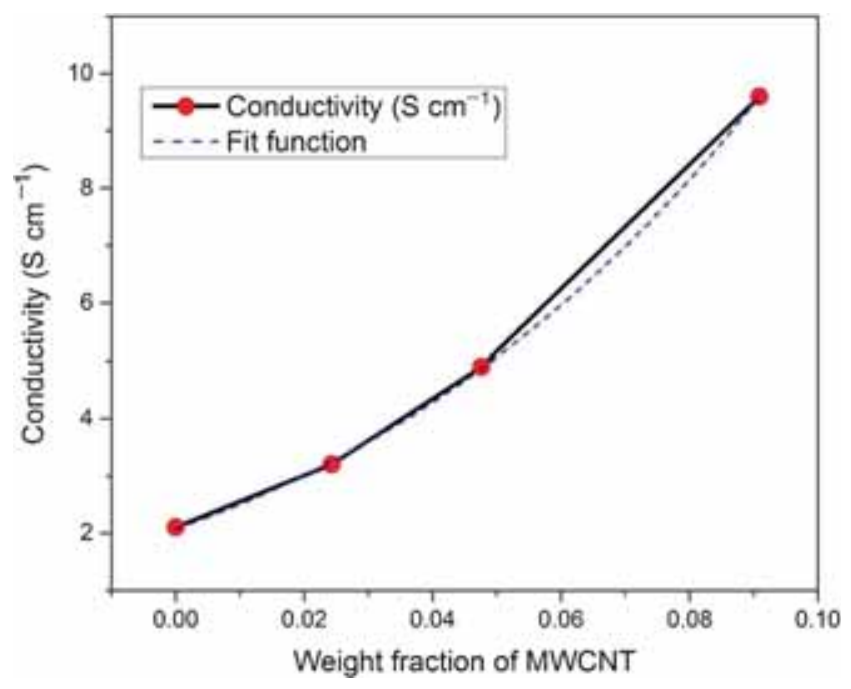

Figure 4. Conductivity plots and percolation threshold function of nanocomposites.

Table 1. Values from figure 4 in percolation threshold equation.

\begin{tabular}{lccc}
\hline Equation & $\sigma_{0}$ & $P_{\mathrm{c}}$ & $t$ \\
\hline$\sigma=\sigma_{0}\left(P-P_{\mathrm{c}}\right)^{t}$ & 394.58 & 0.0402 & 2.21 \\
\hline
\end{tabular}


by absorbing phonons and the conductivity $\sigma$ is expected to follow from path optimization of hopping expanse and difference in energy levels complicated. The temperature dependence of the dc conductivity for conducting polymers tends to follow 3D VRH model's prediction: ${ }^{3,8}$

$$
\sigma=\sigma_{0} \exp \left[-{\frac{T_{\mathrm{mott}}}{T}}^{1 / 4}\right]
$$

where $\sigma_{0}$ and $T_{\text {mott }}$ are the conductivity at infinitive temperature and characteristic temperature, respectively. They are defined as

$$
\begin{aligned}
& \sigma_{0}=e^{2} \vartheta R^{2} N\left(E_{\mathrm{f}}\right), \\
& T_{0}=-\frac{18.1}{\alpha^{3} k_{\mathrm{B}} N\left(E_{\mathrm{f}}\right)},
\end{aligned}
$$

where $e$ is the electronic charge, $\vartheta$ the hopping frequency, $\alpha$ the coefficient of exponential decay of the localized length $(\mathrm{cm}), k_{\mathrm{B}}$ Boltzmann's constant $\left(8.616 \times 10^{-5} \mathrm{eV} \mathrm{K}^{-1}\right)$, $N\left(E_{\mathrm{f}}\right)$ the density of states at the Fermi level $\left(\mathrm{cm}^{-3} \mathrm{eV}^{-1}\right)$. $R$ is the hopping distance $(\mathrm{cm})$ defined as

$$
R=\left[\frac{9}{8 \pi \alpha k_{\mathrm{B}} T N\left(E_{\mathrm{f}}\right)}\right]^{1 / 4} .
$$

Conductivity of PAni and PAni/MWCNT nanocomposites as a function of MWCNT content with increased temperature is shown in figure 5. The relationship between the conductivity of the nanocomposites and MWCNT content was clearly obvious. Also, it can be seen clearly that with growing temperature, conductivity was increased.

The room-temperature conductivity of the PAni doped with p-TSA was $2.1 \mathrm{~S} \mathrm{~cm}^{-1}$, while for $10 \%$ MWCNT nanocomposite it was $9.6 \mathrm{~S} \mathrm{~cm}^{-1}$. To understand the mention around the temperature dependence of dc conductivity of the

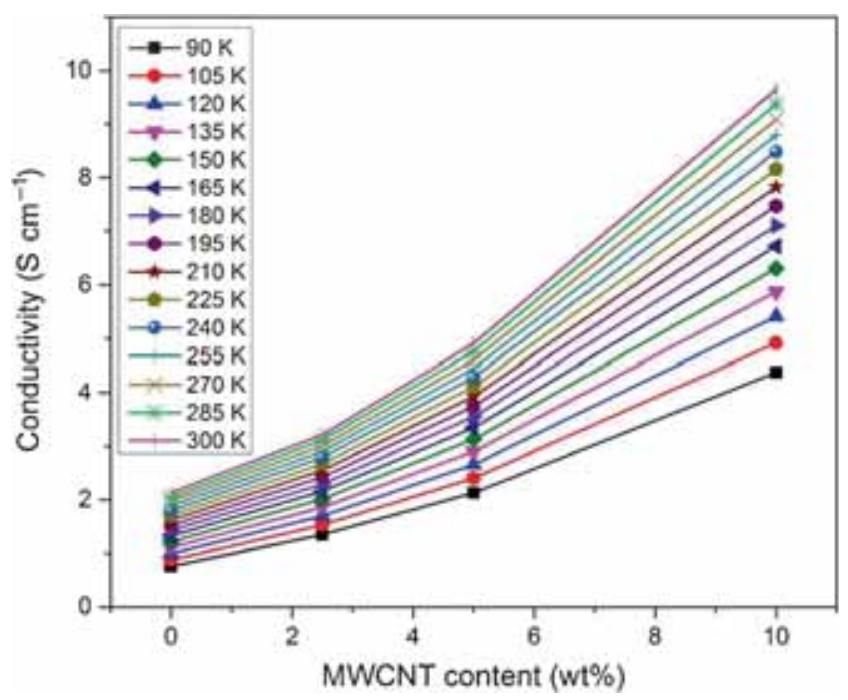

Figure 5. Conductivity of PAni and PAni/MWCNT in various temperatures as a function of MWCNT content.
PAni/MWCNT nanocomposites, the temperature aberration of dc conductivity has been measured contained by a range $90<T<300 \mathrm{~K}$. By plotting $\ln \left(\sigma / \sigma_{0}\right)$ as a function of $T^{-1 / 4}$, a straight line is found (figure 6). The values of the parameters $T_{\text {mott }}$ and $\sigma_{0}$ can be achieved from the slope and from the intersection with the conductivity axis, respectively. The data is shown in table 2.

Polymeric materials consist of bundles of chains arranged parallel to each other. The polymer repeat distance is 4-6 $\AA$ in PAni. ${ }^{24}$ When occupied conjugation is broken we adopt that the polymer chains are divided into conjugated elements of a restricted length. ${ }^{25}$ Therefore, PAni should be treated as a three-dimensional system of long conjugated fragments.

Increasing in conductivity with the combination of MWCNT in the PAni environment is directly related to the existence of conducting MWCNT pathway in the PAni as mentioned before, which indicates percolated behaviour. ${ }^{26}$ PAni acts as a good electron donor however MWCNT are rather good electron acceptors. Consequently, there are some relations between the quinoid rings of PAni and MWCNT which facilitate charge transfer. ${ }^{8}$ Localization length in MWCNT is around $10 \mathrm{~nm}$ due to the presence of a large $\pi$-conjugated structure, therefore, growing the MWCNT content will lead to an increase in the conjugation length of the PAni. ${ }^{27}$ The strong coupling between the MWCNT and PAni improves the average localization length and hence conductivity increases for the nanocomposites.

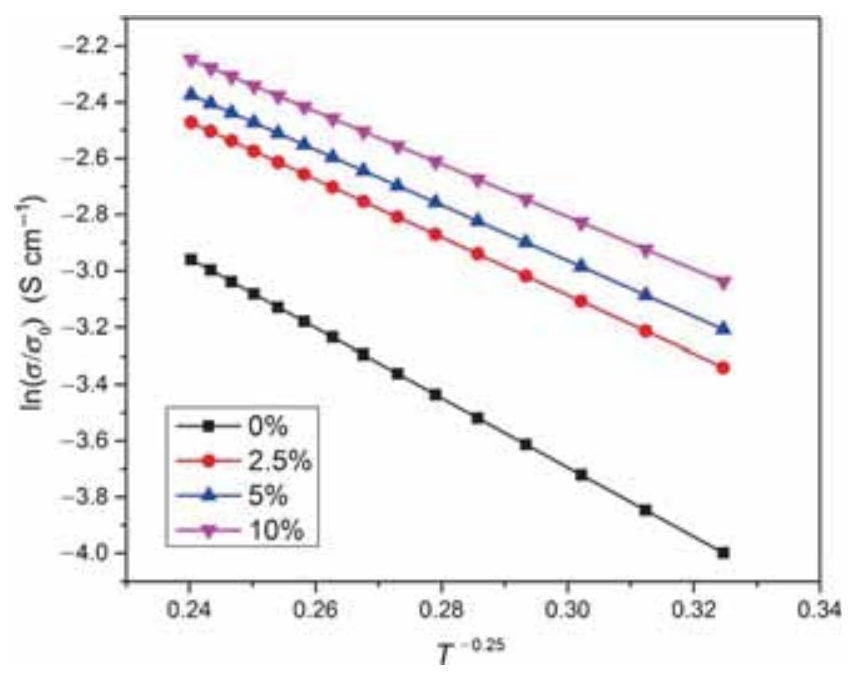

Figure 6. Temperature conductivity dependence of PAni and PAni/MWCNT nanocomposites.

Table 2. $T_{\text {mott }}$ and $\sigma_{0}$ extracted from figure 6 .

\begin{tabular}{lcccc}
\hline Sample & $0 \%$ & $2.5 \%$ & $5 \%$ & $10 \%$ \\
\hline$T_{\text {mott }}$ & $2.3 \times 10^{4}$ & $1.12 \times 10^{4}$ & $9.53 \times 10^{3}$ & $7.67 \times 10^{3}$ \\
$\sigma_{0}$ & 40.44 & 42.83 & 52.61 & 90.89 \\
\hline
\end{tabular}




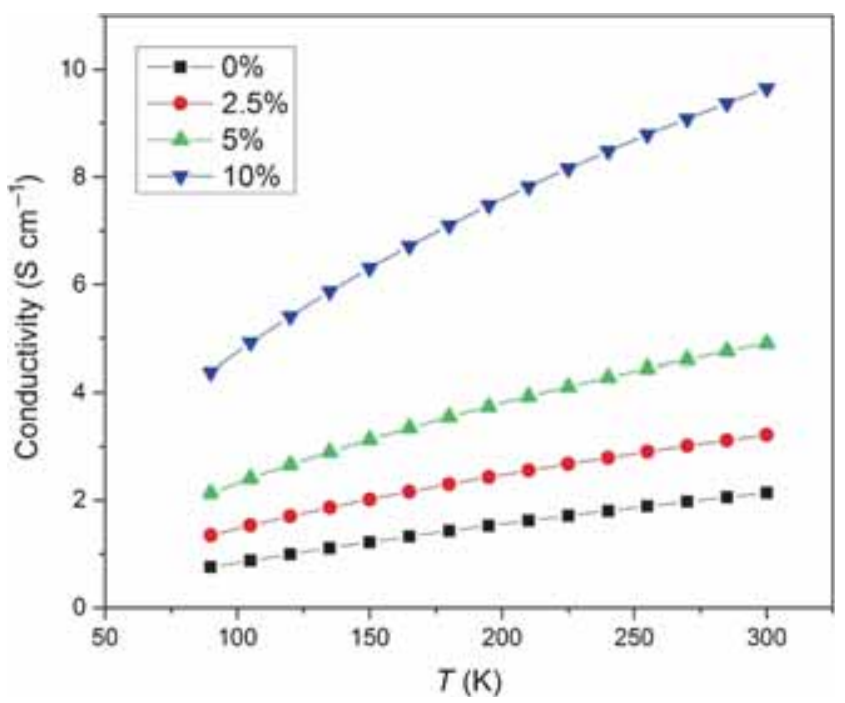

Figure 7. Dependence of conductivity on temperature for PAni and PAni/MWCNT nanocomposites.

The dependence of conductivity on temperature is shown in figure 7.

\section{Conclusion}

PAni/MWCNT nanocomposites have been synthesized successfully via in-situ the chemical oxidative polymerization technique. Structural, morphological and conductivity studies have been performed for PAni and the blend of PAni/MWCNT. The results indicated that PAni and PAni/MWCNT nanocomposites were successfully synthesized by this method. The electrical conductivity of nanocomposites increases with growing wt\% of MWCNT. SEM results confirmed a typically cauliflower-like and orbicular morphology for nanocomposites. Measurement of dc conductivity as a function of temperature has been performed on PAni/MWCNT nanocomposites. The $T^{-1 / 4}$ behaviour of the conductivity, together with practical values achieved for the parameters, confirm transport can follow by 3D VRH. The conductivity temperature dependence experiments showed a strong interaction between the MWCNT and polymer chains which increases the average localization length so that conductivity increases for the nanocomposites. Also, comparable to other semiconductor materials, the carrier density increased with temperature. In-situ oxidative polymerization provides new opportunities for potential applications of these nanocomposites in a number of scientific and technological fields.

\section{Acknowledgements}

We are thankful to Faculty of Materials \& Polymer Engineering, Hakim Sabzevari University, for providing the necessary facilities for the preparation of the paper.

\section{References}

1. Ansari Khalkhali R 2006 E-J. Chem. 3186

2. Ansari R and Keivani M B 2006 E-J. Chem. 3202

3. Campos M and Bello B 1997 J. Phys. D: Appl. Phys. 30 1531

4. Ghadim M F, Imani A and Farzi G 2014 J. Nanostruct. Chem. 41

5. Farzi G and Imani A 2012 Comparison between silicon based and organic based photovoltaic solar cells In: 6th international workshop on crystalline silicon solar cells, Aix-Les-Bains, France

6. Talukdar D, Nandi U N, Bardhan K K, Bof Bufon C C, Heinzel T, De A et al 2011 Phys. Rev. B: Condens. Matter Mater. Phys. 84054205

7. Lin Y F, Chen C H, Xie W J, Yang S H, Hsu C S, Lin M T et al 2012 ACS Nano 51541

8. Imani A, Farzi G and Ltaief A 2013 Int. Nano Lett. 38

9. Chuang F Y and Yang S M 2005 Synth. Met. 152361

10. Abdiryim T, Ubul A, Jamal R and Rahman A 2012 Materials (Basel) 51219

11. Saini P, Choudhary V, Singh B P P, Mathur R B B and Dhawan S K K 2009 Mater. Chem. Phys. 113919

12. Mirmohseni A, Dorraji M S S and Hosseini M G 2012 Electrochim. Acta 70182

13. Das S K and Prakash R 2009 Electrical properties of multiwalled carbon nanotubes polyaniline nanocomposite In: Emerging trends in electronic and photonic devices and systems. ELECTRO '09. international conference. doi: 10.1109/ELECTRO.2009.5441048

14. Ubul A, Jamal R, Rahman A, Awut T, Nurulla I and Abdiryim T 2011 Synth. Met. 1612097

15. McClory C, McNally T, Baxendale M, Pötschke P, Blau W and Ruether M 2010 Eur. Polym. J. 46854

16. Yilmaz F and Küçükyavuz Z 2009 J. Appl. Polym. Sci. 111 680

17. Zengin H, Zhou W, Jin J, Czerw R, Smith D W, Echegoyen L et al 2002 Adv. Mater. 141480

18. Hammersley J M 1957 Math. Proc. Cambridge Philos. Soc. 53 642

19. Christensen K 2002 Percolation Theory 71259

20. Aguilar J O, Bautista-Quijano J R and Avilés F 2010 Express Polym. Lett. 4292

21. Bansal V, Bhandari H, Bansal M C and Dhawan S K 2009 Indian J. Pure Appl. Phys. 47667

22. Foroughi J, Kimiaghalam B, Ghorbani S R, Safaei F and Abolhasan M 2012 Thin Solid Films 5207049

23. Krinichnyi V I, Tokarev S V, Roth H K, Schrödner M and Wessling B 2006 Synth. Met. 1561368

24. Pouget J P, Jozefowicz M E, Epstein A J, Tang X and MacDiarmid A G 1991 Macromolecules 24779

25. Jozefowicz M E, Laversanne R, Javadi H H S, Epstein A J, Pouget J P, Tang X et al 1989 Phys. Rev. B 3912958

26. Long Y, Chen Z, Zhang X, Zhang J and Liu Z 2004 J. Phys. D: Appl. Phys. 371965

27. Chakraborty G, Gupta K, Meikap A K, Babu R and Blau W J 2012 Solid State Commun. 15213 